\section{Gräser-SIT: Synthetische Gräserallergenepitoppeptide klinisch getestet}

\author{
Synthetische Allergenpeptide können die spezifische Immuntherapie \\ möglicherweise weiter verbessern. Noch liegen aber kaum klinische \\ Erfahrungen vor. Eine größere Phase-II-Studie untersuchte Wirksamkeit \\ und Sicherheit von Gräserallergenepitoppeptiden.
}

$S_{\mathrm{re}}^{\mathrm{p}}$ PIREs („synthetic peptide immunoregulatory epitopes") sind eine Weiterentwicklung der klassischen Allergenextrakte. Die T-Zell-Epitop-basierten Peptide bestehen aus acht bis zehn Aminosäuren, zeigen - wie Allergene aus natürlichen Quellen - immunregulatorische Eigenschaften und sollen vor allem die Verträglichkeit und Adhärenz der spezifischen Immuntherapie verbessern. Speziell für die Kurzzeittherapie von Gräserpollenallergien wurde eine Kombination aus sieben synthetischen Einzelpeptiden entwickelt, die die häufigsten Gräserallergen-Sensibilisierungen abdecken. In einer multizentrischen randomisierten Phase-II-PollenkammerStudie standen Wirksamkeit und Verträglichkeit verschiedener Dosisregimes auf dem Prüfstand.
Einbezogen waren 282 erwachsene Gräserpollenallergiker, die nach einer viertägigen standardisierten Basisprovokation in der Pollenkammer die Gräserallergenpeptide intrakutan in drei Dosierungsschemata erhielten: achtmal $6 \mathrm{nmol}$ in zweiwöchigen Abständen $(8 \times 6 \mathrm{Q} 2 \mathrm{~W})$, viermal $12 \mathrm{nmol}$ in vierwöchigen Intervallen $(4 \times 12 \mathrm{Q} 4 \mathrm{~W})$ oder achtmal $12 \mathrm{nmol}$ in zweiwöchigen Intervallen $(8 \times 12 \mathrm{Q} 2 \mathrm{~W})$ oder Placebo. Die Injektionen wurden jeweils präsaisonal gegeben. Primäres Studienziel war die Veränderung des Gesamt-Rhinokonjunktivitis-Symptomscores bei experimenteller Pollenexposition zu Studienbeginn und den posttherapeutischen Expositionstagen 2, 3 und 4.

Die größten Scoreveränderungen im Vergleich zu Placebo ergaben sich in der
$8 \times 6 \mathrm{Q} 2 \mathrm{~W}$-Gruppe mit $-5,4$ vs. $-3,8$ (p = $0,346)$. In der $8 \times 6 \mathrm{Q} 2 \mathrm{~W}-\mathrm{Gruppe}$ zeigten Patienten mit einer stärker ausgeprägten Rhinokonjunktivitis-Symptomatik eine signifikante Symptomverbesserung im Vergleich zu Placebo ( $p=0,0403)$. Die Inzidenzen von Unverträglichkeiten waren in den drei Therapiegruppen und der Placebogruppe vergleichbar, untypische schwere Reaktionen wurden nicht gesehen. Injektionsreaktionen traten unter den Peptiden mit Raten zwischen 7-17\% vergleichsweise selten auf, lagen aber deutlich über denen von Placebo mit $1 \%$.

Fazit: Eine SPIRE-Kurzzeittherapie bei Patienten mit einer gräserallergenbedingten Rhinokonjunktivitis ist klinisch relevant wirksam und sicher, so das Ergebnis einer Pollenkammerstudie. Dabei zeigte die achtmalige präsaisonale intrakutane Injektion einer mittelhohen kumulativen Dosis alle zwei Wochen insgesamt das beste Nutzen-RisikoVerhältnis.

Dr. Barbara Kreutzkamp

Ellis AK et al. Treatment with grass allergen peptides improves symptoms of grass polleninduced allergic rhinoconjunctivitis. J Allergy Clin Immunol 2017; 140: 486-96

\section{Atopische Dermatitis: Erkrankungsalter verrät Allergierisiko}

\begin{abstract}
Die atopische Dermatitis kann bei Kindern offenbar in Abhängigkeit des Erkrankungsalters und des Symptomenverlaufs in vier verschiedene Untergruppen unterteilt werden. Dabei zeigen sich vor allem hinsichtlich der Wahrscheinlichkeit eines Etagenwechsels große gruppenspezifische Unterschiede.
\end{abstract}

\begin{abstract}
$\mathrm{n}$ den Industriestaaten leiden mehr als $20 \%$ der Kinder an atopischer Dermatitis (AD). Bei über $60 \%$ treten die ersten Symptome bereits vor dem zweiten Lebensjahr auf. Zwar „wächst“ sich bei einem Teil der kleinen Patienten die Problematik aus, doch gilt die AD als erstes Indiz für eine atopische Veranlagung. Gefürchtet ist in diesem Zusammenhang vor allem ein späterer Etagenwechsel hin zum allergischen Asthma oder einer Nahrungsmittelallergie. Doch auch wenn der Zusammenhang zwi-
\end{abstract}

schen $\mathrm{AD}$, Asthma und Heuschnupfen eindeutig nachgewiesen ist, ist man mittlerweile von der Vorstellung einer einheitlichen Allergikerkarriere abgerückt. Mittlerweile geht man vielmehr davon aus, dass das Risiko für weitere Allergien in Abhängigkeit von Manifestationszeitpunkt und Verlauf der AD deutlich variiert. Entsprechend hat man sich daran gemacht, Untergruppen zu identifizieren.

Kinderärzte aus der Schweiz haben dazu auf die Daten der PASTURE
(Protection against Allergy Study in Rural Enviroments)-Studie zurückgegriffen, einer großen europaweiten Studie, in der nach Faktoren gefahndet wurde, die eine $\mathrm{AD}$ bei Kindern begünstigen oder verhindern helfen.

Tatsächlich konnten anhand der Daten von 1.038 Kindern im Rahmen einer Latent-Class-Analyse vier phänotypische Subgruppen definiert werden. Kinder mit einem frühen transienten Phänotyp ( $n=96 ; 9,2 \%)$ entwickeln bereits innerhalb der ersten zwei Lebensjahre AD-Symptome, sind jedoch nach dem vierten Geburtstag wieder völlig beschwerdefrei. Kinder mit einem frühen persistierenden Phänotyp ( $n=67 ; 6,5 \%$ ) erkranken zwar ebenfalls sehr früh, die AD bleibt allerdings bis zum Alter von sechs Jahren bestehen. Kinder, deren AD dem späten Phänotyp zuzuordnen ist $(\mathrm{n}=50 ; 4,8 \%)$, entwickeln erst nach dem zweiten Lebensjahr die ersten Hautsymptome. Beim sporadischen Phänotyp 